\title{
Vascular matrix remodeling in patients with bicuspid aortic valve malformations: Implications for aortic dilatation
}

\author{
Paul W. M. Fedak, MD \\ Mauro P. L. de Sa, MD, PhD \\ Subodh Verma, MD, PhD ${ }^{a}$ \\ Nafiseh Nili, $\mathrm{PhD}^{\mathrm{c}}$ \\ Pedram Kazemian, $\mathrm{MSc}^{\mathrm{a}}$ \\ Jagdish Butany, $\mathrm{MD}^{\mathrm{b}}$ \\ Bradley H. Strauss, MD, $\mathrm{PhD}^{\mathrm{c}}$ \\ Richard D. Weisel, MD \\ Tirone E. David, MD
}

\footnotetext{
From the Division of Cardiac Surgery and the Department of Pathology, ${ }^{\mathrm{b}}$ University of Toronto, Toronto General Hospital, University Health Network, Toronto, Ontario, Canada, and the Division of Cardiology, ${ }^{\mathrm{c}}$ University of Toronto, Roy and Ann Foss Research Program, Terrence Donnelly Heart Centre, St Michael's Hospital, Toronto, Ontario, Canada.

Read at the C. Walton Lillehei Resident Forum of the Eighty-second Annual Meeting of The American Association for Thoracic Surgery, Washington, DC, May 5-8, 2002.

P.W.M.F. is a Research Fellow of the Canadian Institute for Health Research (CIHR) and the Heart and Stroke Foundation of Canada (HSFC).

Received for publication June 5, 2002; revisions requested Sept 3, 2002; revisions received Jan 26, 2003; accepted for publication Feb 11, 2003.

Address for reprints: Tirone E. David, MD, Division of Cardiac Surgery, Toronto General Hospital, 13EN-219, 200 Elizabeth St, Toronto, Ontario, Canada M5G 2C4 (Email: tirone.david@uhn.on.ca).

J Thorac Cardiovasc Surg 2003;126: 797-806

Copyright (C) 2003 by The American Association for Thoracic Surgery

$0022-5223 / 2003 \$ 30.00+0$

doi:10.1016/S0022-5223(03)00398-2
}

Background: Patients with bicuspid aortic valve malformations are at an increased risk of aortic dilatation, aneurysm formation, and dissection. Vascular tissues with deficient fibrillin-1 microfibrils release matrix metalloproteinases, enzymes that weaken the vessel wall by degrading elastic matrix components. In bicuspid aortic valve disease a deficiency of fibrillin-1 and increased matrix metalloproteinase matrix degradation might result in aortic degeneration and dilatation.

Methods: Samples of the pulmonary artery and aorta were obtained from surgical patients with bicuspid aortic valves $(n=21)$ and tricuspid aortic valves $(n=16)$.

Results: Fibrillin-1 content was reduced in bicuspid aortic valve aortas compared with that seen in tricuspid aortic valve aortas $(P=.001)$, whereas the associated matrix components, elastin and collagen, were unchanged $(P=.51$ and $P=.21)$. Reductions of aortic fibrillin-1 content were independent of valve function and patient age. Compared with tricuspid aortic valve aorta, matrix metalloproteinase 2 activity was increased more than 2-fold in bicuspid aortic valve aortas $(P=.04)$ and correlated positively with aortic diameter $(r=0.74, P=.05)$. Matrix metalloproteinase 9 activity was not significantly different. Fibrillin- 1 content was also reduced in the pulmonary arteries of patients with bicuspid aortic valves $(P=.06)$, suggesting a systemic deficiency of fibrillin-1. Promatrix metalloproteinase 2 was increased $(P=.04)$, reflecting an increased production of matrix metalloproteinase 2 in these fibrillin-1-deficient tissues, whereas active matrix metalloproteinase 2 and matrix metalloproteinase 9 species were unchanged, and correspondingly, the pulmonary arteries were not dilated.

Conclusions: Deficient fibrillin-1 content in the vasculature of patients with bicuspid aortic valves might trigger matrix metalloproteinase production, leading to matrix disruption and dilatation. This process of vascular matrix remodeling in patients with bicuspid aortic valves offers novel therapeutic targets to prevent the aortic degeneration and dilatation characteristic of this disease.

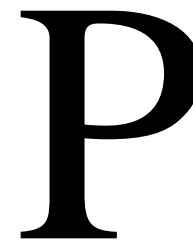
atients with bicuspid aortic valve (BAV) malformations, independent of valvular function, are at an increased risk of aortic dilatation, aneurysm, and dissection. ${ }^{1}$ Given the high prevalence of this anomaly, the surgical management of these patients is frequent. A common underlying genetic basis has been considered for the associated defects in both aortic valvular and vascular structure, but supporting evidence has been lacking. The cellular and molecular mechanisms that lead to these serious aortic complications remain elusive. 
Fibrillin-1 is a fundamental extracellular matrix component of the aortic media. ${ }^{2}$ The fibrillin-rich microfibrils play a prominent role in regulating tissue development and maintaining tissue elasticity by linking vascular smooth muscle cells to adjacent elastin fibrils. ${ }^{3}$ Gene defects that alter fibrillin-1 content, as in Marfan syndrome and other disorders, can reduce the structural integrity of the vessel wall, leading to aortic dilatation or dissection. ${ }^{4}$ In transgenic mouse models targeted underexpression of the fibrillin-1 gene results in aortic aneurysms with increased matrix metalloproteinase (MMP) matrix fragmentation, reducing the structural integrity of the aorta. ${ }^{3}$ In patients with aortic valve malformations, we and others have documented medial degeneration ${ }^{5}$ consistent with that seen in experimental models of fibrillin-1 deficiency, suggesting that a similar pathologic process might occur.

In the present study we hypothesized that BAV disease is associated with a fibrillin-1 deficiency and increased MMP activity.

\section{Methods}

\section{Sample Collection}

Samples of the aorta and pulmonary artery were collected during cardiac surgery from 21 patients with congenital BAV malformations, including 2 patients with grossly malformed cusps consistent with the unicuspid aortic valve condition. For comparison, samples of the aorta and pulmonary artery were collected during cardiac surgery from 16 patients with tricuspid aortic valves (TAVs). Of these 37 patient samples, matched aorta and pulmonary artery specimens from the same patient were obtained in 22 patients $(\mathrm{BAV}, \mathrm{n}=11 ; \mathrm{TAV}, \mathrm{n}=11)$. Tissue samples approximately 1.0 $\mathrm{cm}^{2}$ in size were obtained $1 \mathrm{~cm}$ distal to the sinotubular junction from the anterior aspect of the proximal ascending aorta and main pulmonary artery. Samples were promptly embedded in OCT freezing compound (Tissue-Tek 4583), snap-frozen in liquid nitrogen, and stored at $-70^{\circ} \mathrm{C}$ until use. Patient consent and ethics approval was obtained from the Research Ethics Board, University Health Network, before sample collection.

\section{Immunohistochemistry}

Samples were fixed in acetone, cryosectioned at a 4- to 5- $\mu \mathrm{m}$ thickness, and air-dried. To expose elastin-associated fibrillin-1 and remove elastin autofluorescence, which could mask the fluorescence of fibrillin-1, enzymatic digestion of the sections before fibrillin-1 staining was performed by incubating the samples in $0.01 \%$ elastase (Worthington Biochemical) in Tris- $\mathrm{HCl}$ buffer (67 $\mathrm{mmol} / \mathrm{L}, \mathrm{pH} 8.8$ ) for 20 minutes. Sections were stained for fibrillin-1 with mouse anti-fibrillin monoclonal antibody (clone 12A5.18, Neomarkers Inc) at a 1:50 dilution for 1 hour. For elastin staining, samples were air-dried, formalin fixed, and incubated in rabbit polyclonal anti- $\alpha$-elastin antibody (Neomarkers Inc) at 1:100 dilution for 1 hour. For all sections, goat anti-mouse $\operatorname{IgG}$ conjugated to Cy3 (Jackson Immunoresearch Lab, Inc) was used as a secondary antibody. All sections were counterstained for DNA with 4,6 diamino-1-phenylindolel (Sigma Chemical Co). Sections were mounted and examined with a fluorescence microscope. Negative controls were stained, omitting the incubation step with the primary antibody.

\section{Quantitative Fluorescence Microscopy}

For quantification of immunostaining, the indirect immunofluorescence method was used with a MicroComputer Image Device (MCID-M2, Imaging Research Inc) mounted on an epi-illumination fluorescence microscope (Olympus BX50, Olympus America Inc). The secondary antibody, $\mathrm{Cy} 3$, was excited with an excitation filter (515-530 nm of green light), and the emission was detected by using an emission filter (540 and $570 \mathrm{~nm}$ ). Microscopic fields of view showing the full thickness of the vessel wall were displayed on the computer monitor. Regions measuring $200 \times 300$ $\mu \mathrm{m}$ with the $10 \times$ objective for fibrillin- 1 and $100 \times 150 \mu \mathrm{m}$ with the $20 \times$ objective for elastin were selected for quantitative analysis. Nine different regions of each specimen, 3 in each of the inner, middle, and outer portions of the media, were quantitatively analyzed. A negative control was used for each slide to account for any background fluorescence resulting from autofluorescence or any nonspecific binding of the Cy3-conjugated secondary antibody. The fluorescence level was quantified by measuring the integrated optical density (IOD) of each sample and its negative control according to a method described by Godfrey and colleagues. ${ }^{6}$

The IOD of each image was measured by using a histogram of the cumulative relative percentages of the gray scale score. Gray scale score is a value assigned to each pixel of the image on the basis of its relative brightness level. The nonfluorescence background is zero, and any pixel with a nonzero score represents fluorescence. The IOD is obtained by subtracting the background percentage from $100 \%$. The fluorescence level of each sample (average of 9 different regions), represented by mean IOD, was calculated by subtracting the mean IOD of the negative control from that of the sample labeled with primary antibody. Fibrillin-1 slides were also reanalyzed by using the $20 \times$ objective to determine whether the magnification could interfere with results. No difference was observed between results obtained with the $10 \times$ or $20 \times$ objective. An observer blinded to the groups performed all assessments.

\section{Hydroxyproline Determination of Collagen and Elastin} Collagen and elastin content were also determined by using the method of Strauss and colleagues. ${ }^{7}$ Samples were digested overnight by using a cyanogen bromide treatment $(50 \mathrm{mg} / \mathrm{mL}$ in $70 \%$ formic acid), which solubilizes all proteins except elastin by cleaving methionine bonds. The supernatant, containing fragments of collagen and other proteins, was dried and hydrolyzed in $6 \mathrm{~N} \mathrm{HCl}$ at $110^{\circ} \mathrm{C}$ for 24 hours. Because collagen is the only protein in the supernatant fraction containing significant amounts of hydroxyproline, collagen content was measured by determining the total hydroxyproline content of the hydrolysate. The insoluble residue after cyanogen bromide treatment contains essentially pure elastin on the basis of amino acid analysis. ${ }^{8}$ As such, the dry weight of the residue was taken as the content of total insoluble elastin in the vascular tissue. 


\section{Gelatinase Zymography}

Samples were pulverized in liquid nitrogen and extracted in icecold extraction buffer (cacodylic acid, $10 \mathrm{mmol} / \mathrm{L}$; $\mathrm{NaCl}, 0.15$ $\mathrm{mol} / \mathrm{L} ; \mathrm{ZnCl}_{2}, 20 \mathrm{mmol} / \mathrm{L} ; \mathrm{NaN}_{3}, 1.5 \mathrm{mmol} / \mathrm{L}$; and $1 \%$ sodium dodecylsulfate, $\mathrm{pH}$ 5.0). After 1 hour, the supernatant was separated from any insoluble debris by means of centrifugation $\left(4^{\circ} \mathrm{C}\right.$, 10 minutes, 13,000 rpm) and assayed for protein concentration by using the Bio-Rad protein assay kit (Bio-Rad). Samples were placed in aliquots and stored at $-80^{\circ} \mathrm{C}$ until use. Gelatin zymography was performed according to the method of Cruz and colleagues. ${ }^{9}$ Extracts containing $40 \mu \mathrm{g}$ of protein were loaded onto a $10 \%$ sodium dodecylsulfate-polyacrylamide gel precast with $0.1 \%$ gelatin substrate (Criterion Zymogram Gels, Bio-Rad) and electrophoresed at $125 \mathrm{~V}$. After electrophoresis, the gels were washed in $2.5 \%$ Triton $\mathrm{X}-100$, incubated overnight at $37^{\circ} \mathrm{C}$ in Tris buffer (Tris, $50 \mathrm{mmol} / \mathrm{L} ; \mathrm{CaCl}_{2}, 15 \mathrm{mmol} / \mathrm{L}$ ), and then stained with $0.5 \%$ Coomassie brilliant blue R-250 (Bio-Rad). After destaining for 4 hours, areas of clearing in the gels indicated the presence of gelatinase activity (MMP-2 and MMP-9). Gels were calibrated with molecular weight markers (Bio-Rad) and positive controls (activated purified MMP-2 and MMP-9). The zymograms were digitized, and MMP activity was determined by means of quantitative image analysis (Scion Image, NIH Software).

\section{Statistical Analysis}

Unless otherwise specified, all variables are reported as means \pm SD. Differences between groups were evaluated by means of 1-way analysis of variance. The post hoc Bonferroni multiple comparison test was used to find differences between groups. Categoric variables were analyzed by using the $t$ test or the Fisher exact test when numbers were small.

\section{Results}

\section{Patient Characteristics}

In the BAV group 12 patients had aortic stenosis, and 9 had aortic regurgitation. In the TAV group 5 patients had aortic stenosis and 5 had aortic regurgitation, and 6 patients undergoing heart transplantation had TAVs free of disease. The average age was $42 \pm 12$ years for the BAV group and $50 \pm 17$ years for the TAV group. Preoperative echocardiography revealed aortic dilatation (defined as $>40 \mathrm{~mm}$ in diameter) in 10 of the patients with BAVs $(40,43,43,44$, $48,48,48,52,53$, and $55 \mathrm{~mm}$ ) and in 1 patient with a TAV $(70 \mathrm{~mm})$. The main pulmonary artery was not dilated in any of the patients studied.

\section{Fibrillin-1 Content}

The mean IOD for aortic fibrillin-1 content was not different in patients with normal or diseased aortic valves $(26.0 \pm 5$ vs $20.6 \pm 9, P=.45$ ). Because values in patients with nondiseased TAVs were not statistically different from those in patients with diseased TAVs, all patients with TAVs were combined for comparison with the BAV group. Patients with BAVs had significantly less fibrillin-1 than patients with TAVs (mean IOD \pm SD: $14.1 \pm 8$ vs $24.5 \pm$ $7, P=.001$; Figure 1, A). This difference was uniform
A
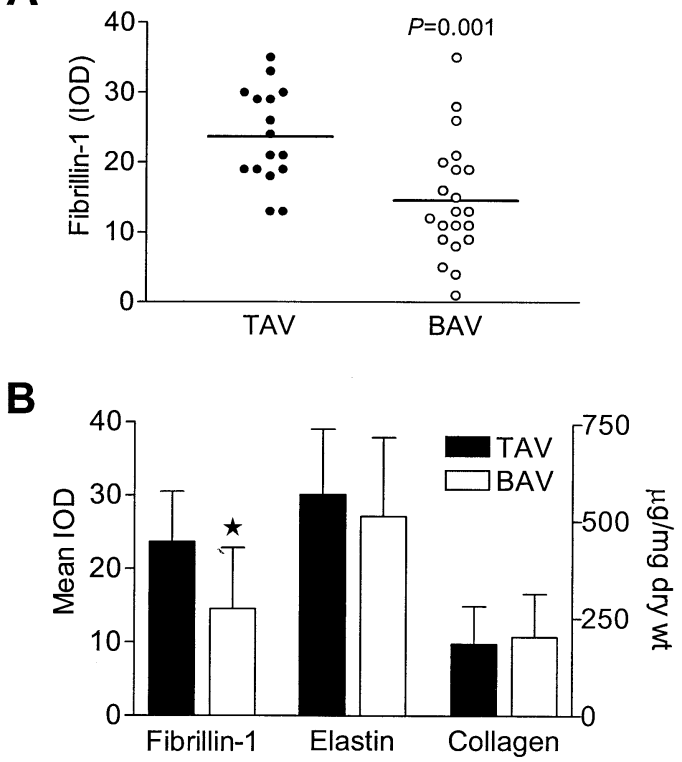

Figure 1. A, As determined by means of indirect immunofluorescence, patients with BAV disease had significantly less fibrillin-1 in the aortic media than did patients with TAVs $(P=.02)$, whereas elastin and collagen contents were similar (B). Bars represent group means \pm SD. ${ }^{*} P<.05$.

because the mean IOD in the inner, middle, and outer portions of the media was not statistically different. Linear regression analysis did not show a relationship between fibrillin-1 content and either aortic diameter (BAV and TAV: $P=.58$ and $P=.17$, respectively) or patient age (BAV and TAV: $P=.84$ and $P=.12$, respectively).

\section{Elastin and Collagen Content}

As determined by means of immunofluorescence, elastin content was not different between the BAV and TAV groups (mean IOD \pm SD: $36.3 \pm 14$ vs $32.7 \pm 19, P=.6$ ). In addition, mean elastin content, by determining the insoluble fraction after cyanogen bromide digestion, was not significantly different between the BAV and TAV groups $(569 \pm 169$ vs $513 \pm 203 \mu \mathrm{g} / \mathrm{mg}$ dry weight, $P=.51$; Figure $1, B)$. The mean IODs of aortic elastin in patients with normal and diseased valves were not significantly different $(31.8 \pm 12$ vs $33.2 \pm 23, P=.9)$. There was no statistically significant linear correlation between the elastin content and either aortic diameter (BAV and TAV: $P=.9$ and $P=.27$, respectively) or patient age (BAV and TAV: $P=.15$ and $P=.77$, respectively).

Collagen content, as assessed by means of hydroxyproline determination, was not significantly different between the BAV and TAV groups $(203 \pm 111 \mathrm{vs} 185 \pm 97 \mu \mathrm{g} / \mathrm{mg}$ dry weight, $P=.21$; Figure $1, B$ ). 

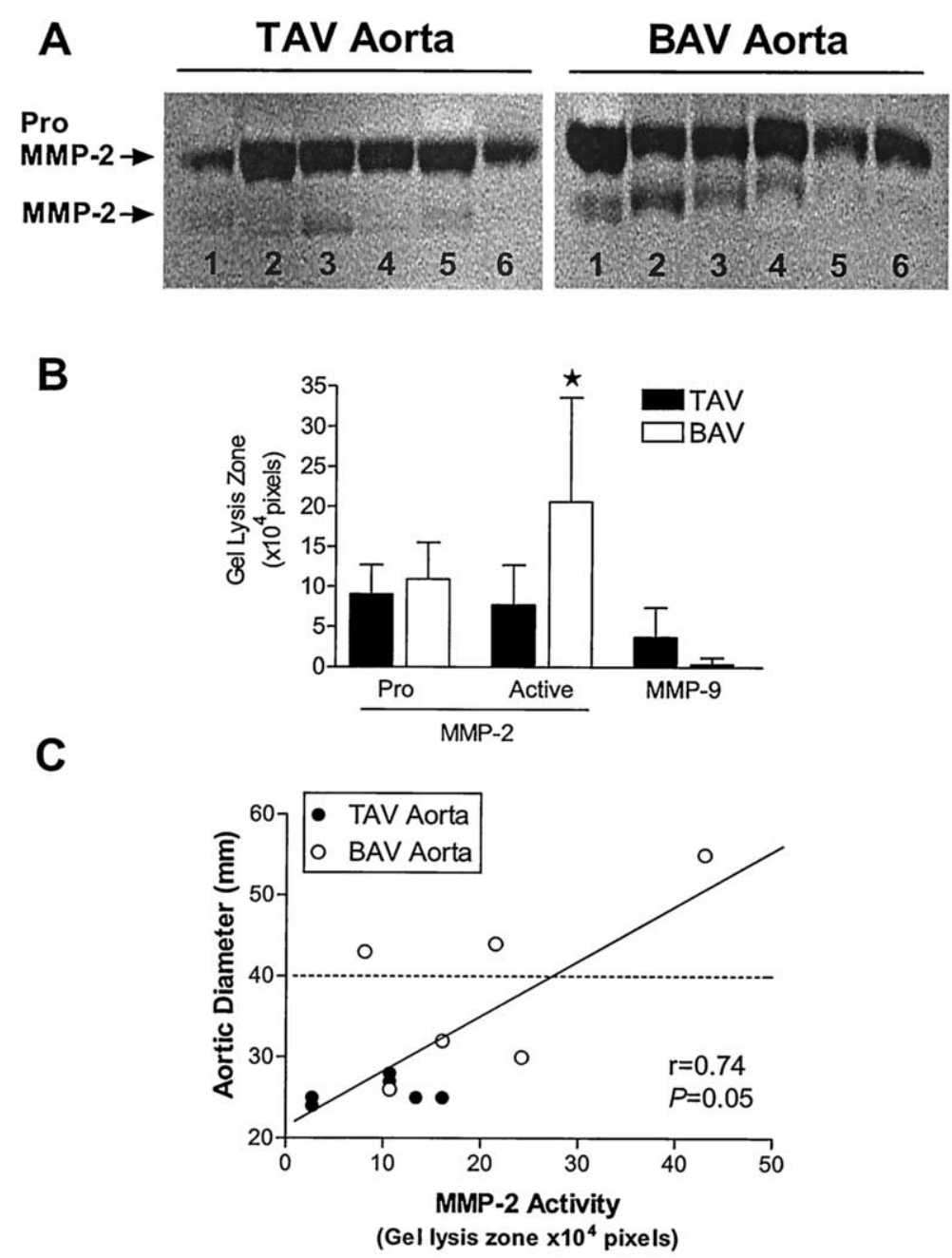

Figure 2. A, MMP gelatin zymogram indicating increased active MMP-2 in the aorta of patients with BAVs compared with that in patients with TAVs. B, Compared with aorta from patients with TAVs, MMP-2 activity was increased more than 2-fold in patients with BAV disease (mean densitometric units \pm SD: $20.6 \pm 13$ vs $7.7 \pm 6$, $\boldsymbol{P}=.04)$. Bars represent group means \pm SD. ${ }^{*} \boldsymbol{P}<.05$. C, Increased MMP activity might disrupt vessel wall integrity, given that MMP-2 activity positively correlated with aortic dilatation $(r=0.74, P=.05)$.

\section{MMP Activity}

Compared with TAV aortas, MMP-2 activity was increased over 2-fold in BAV aortas (mean densitometric units: 20.6 \pm 13 vs $7.7 \pm 5, P=.04)$ and correlated positively with aortic diameter $(r=0.74, P=.04$, Figure 2$)$. Pro-MMP-2 and MMP-9 levels were not significantly different (Figure 2, $B)$.

\section{Pulmonary Artery Analysis}

Pulmonary arterial samples were similarly assessed to determine whether reductions of fibrillin-1 and increased MMP-2 activity were systemic. Comparable with their matched aortic samples, the pulmonary artery showed similar reductions of fibrillin-1 content (Figure 3, $A$ and $B$ ).
Pro-MMP-2 activity was significantly increased, but active MMP-2 and MMP-9 were not significantly different (Figure $3, C)$. These data indicate an increased production of MMP-2 without corresponding increases in its activity. In contrast to the aorta samples, none of the pulmonary artery samples had increased active MMP species, and correspondingly, none of the pulmonary arteries had dilatation on echocardiography.

\section{Discussion}

The present study was aimed at understanding the mechanisms of aortic degeneration and dilatation in patients with BAV malformations. BAVs are frequently associated with significant aortic pathology, ${ }^{1}$ and a common underlying 
congenital cause has been proposed. Although this idea is appealing, definitive evidence to support this concept has been lacking. In these patients we and others have demonstrated characteristic degenerative lesions within the vessel wall. ${ }^{1,10,11}$ As in the Marfan aorta, the aortic media in patients with BAV malformations has extracellular matrix fragmentation adjacent to areas of smooth muscle cell necrosis. The mechanisms underlying the remodeling of vascular matrix in patients with BAV disease and the association of these changes with aortic dilatation is unclear.

Advances in molecular biology offer new insights into the pathogenesis of aortic dilatation resulting from molecular defects in genes that encode for matrix elements. Analysis of varied combinations of normal and mutant fibrillin-1 gene expression points to a threshold phenomenon for the deterioration of the vessel wall that is based on the abundance and integrity of fibrillin-1 microfibrils. ${ }^{12}$ Mice homozygous for a targeted hypomorphic allele of the fibrillin-1 gene have structurally normal fibrillin-1 protein but at a significantly reduced level. In these mouse models fibrillin-1 protein deficiency resulted in increased MMP matrix fragmentation, reducing the structural integrity of the aorta. Disruption of the aortic media resulted in progressive aortic dilatation. ${ }^{13}$ Reduced fibrillin-rich microfibrils dissociated smooth muscle cells from elastic laminae and stimulated the cells to increase constitutive MMP activity and undergo apoptotic cell death. ${ }^{14}$ Premature, medial-layer, smooth muscle cell apoptosis has been associated with aortic valve malformations. ${ }^{15}$ The pathology of the aorta in these experimental models of fibrillin-1 deficiency resembles that in patients with congenital aortic valve malformations, which encouraged us to explore a similar pathophysiologic mechanism in our patients with BAVs.

The present study outlines a mechanism of vascular matrix remodeling that might contribute to the aortic pathology associated with BAV malformations. We hypothesized that the process of vascular matrix reorganization in patients with BAVs was similar to those identified in experimental models of fibrillin-1 deficiency, ${ }^{10}$ and as such, we assessed the abundance of key matrix components in the vasculature of these patients. Our data indicates that a subset of patients with congenital BAV disease have significantly reduced fibrillin-1 content in the aortic media (Figure 1, A). These fibrillin-1 reductions occurred without comparable reductions in the matrix components elastin and collagen (Figure 1, B). Fibrillin-1 content was equivalent in the aorta of patients with normal and diseased TAVs, suggesting that reductions of fibrillin- 1 are not caused by valve dysfunction and are specific to the presence of a congenital BAV malformation, irrespective of its function. In addition, this novel observation is not typical of diseased aorta; fibrillin-1 content is increased, not reduced, in atherosclerotic aortic dilatation. ${ }^{16}$

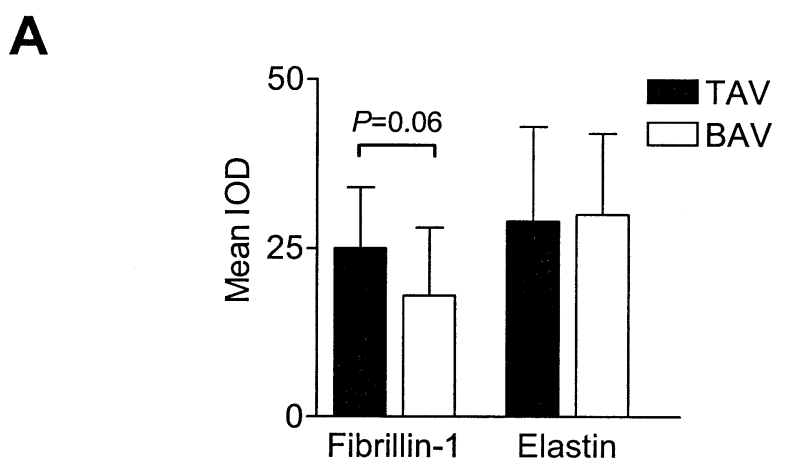

B

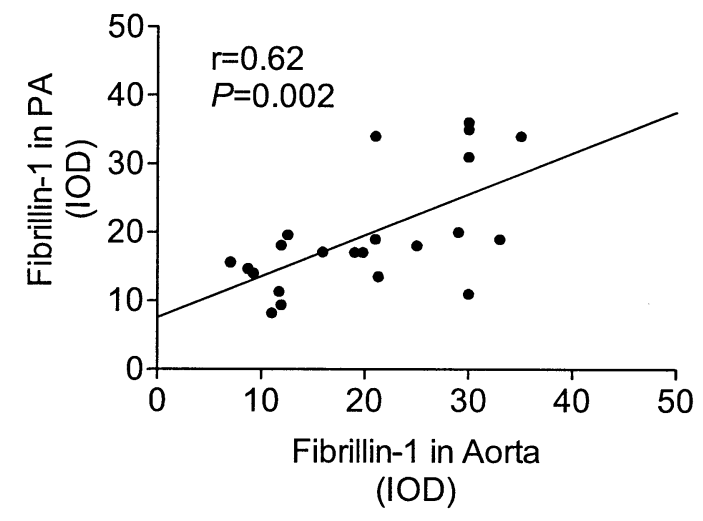

C
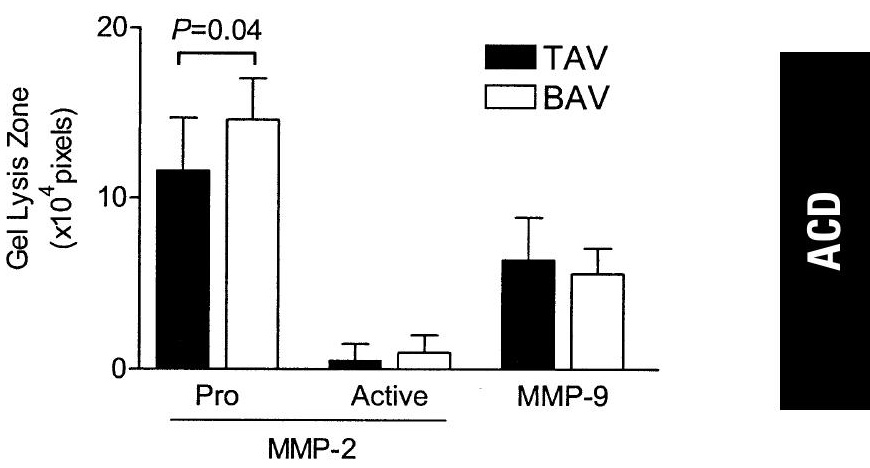

Figure 3. A, As determined by indirect immunofluorescence, fibrillin-1 was reduced in the pulmonary arteries of patients with BAVs $(P=.06)$, whereas its companion matrix component, elastin, was unchanged. Similar to the aorta, these data suggest a systemic deficiency of fibrillin-1 in patients with BAVs, perhaps from aberrant regulation of the genes that encode and process the fibrillin-1 protein into an intact matrix component. B, The deficiency of fibrillin- 1 was heterogeneous between patients but consistent in matched aorta and pulmonary artery samples from the same patients. These data indicate a systemic deficiency of fibrillin-1 in some patients with BAV disease. C, As determined by means of MMP gelatin zymography, pro-MMP-2 was significantly increased in the pulmonary arteries of patients with BAVs, indicating increased production of MMP-2. However, active MMP-2 and MMP-9 were not different from that seen in TAV pulmonary arteries, and correspondingly, the pulmonary arteries were free from dilatation. Bars represent group means \pm SD. 
Given that matrix-degrading enzymes do not act on fibrillin-1 independently, ${ }^{17}$ the fibrillin-1 deficiency might be a result of reduced fibrillin-1 protein production, a failure of compensatory replacement of lost fibrillin-1 in the face of ongoing matrix degradation, or both. Although our observations are suggestive of aberrant gene regulation, it is unclear whether these changes are due to a primary failure in the regulation of the gene, which would occur systemically, or excessive degradation and inadequate replacement of this protein specific to the diseased aorta. To further investigate this mechanism, we assessed matched pulmonary artery samples in patients with TAVs versus BAVs for matrix content. In subsets of patients with BAV malformations, a pathologic deficiency of fibrillin-1 was also observed in the pulmonary artery. These data indicate that fibrillin-1 might be deficient systemically in these patients, which is most consistent with dysregulation of the fibrillin-1 gene. However, our data are limited to the level of the protein and cannot discriminate between a primary defect in expression of the fibrillin- 1 gene versus a secondary effect from inadequate processing and deposition of fibrillin-1 into the extracellular space or excessive fibrillin-1 degradation with inadequate compensatory replacement.

In patients with BAV malformations, the possibility of a molecular defect in genes that regulate the production and assembly of microfibrillar proteins, such as fibrillin-1, is intriguing. Mutations in the fibrillin-1 gene itself that result in decreased gene expression have been associated with thoracic aortic aneurysms and dissection in the absence of Marfan syndrome, ${ }^{4,18}$ although they have yet to be described in families with BAVs. In theory, altered fibrillin-1 gene and protein structure would result in the Marfan syndrome or similar conditions. A reduction in fibrillin- 1 abundance, with preserved protein structure, could result in a similar but less severe phenotype restricted to the vasculature. However, the lack of skeletal manifestations in patients with BAVs, like those of patients with Marfan syndrome, argues against an inherited and systemic deficiency of fibrillin-1, whether caused by a primary gene defect or secondary microfibrillar losses. For example, patients with inherited defects that reduce the systemic expression of fibrillin-1, such as the MASS phenotype and familial Marfanoid habitus, characteristically show prominent abnormalities in the skeleton and skin, with only mild aortic disease. On the other hand, the relationship of fibrillin-1 defects and their diverse and variable clinical manifestations is not well understood. Marfan syndrome and its related phenotypes (ie, MASS phenotype) have a highly variable timing of onset, tissue distribution (ie, vascular vs skeletal and proximal vs distal ascending aorta), and severity of clinical manifestations, even in patients with identical fibrillin- 1 gene mutations and expression levels. ${ }^{19}$ Thus the cause of fibrillin-1 deficiency in patients with BAV malformations remains an open question.

It has been suggested that a common genetic defect underlies both the valvular and vascular complications of BAV disease. Is fibrillin-1 the elusive BAV gene? The microfibrillar proteins, such as fibrillin-1, act as scaffolding for embryonic cells and aid in the formation of both the aortic valve and aorta. ${ }^{20,21}$ In fact, differentiation of cushion mesenchymal cells into mature valve cells correlates with the expression of fibrillin- $1 .^{21}$ Inadequate production or excessive degradation of fibrillin-1 during valvulogenesis might disrupt the formation of the aortic cusps, resulting in a bicuspid valve, as well as a weakened aorta. Yet in multiple clinical states associated with defects in the structure, expression, or both of fibrillin-1, such as the Marfan and MASS phenotypes, there does not appear to be an increased propensity for congenital aortic valve malformations. The possibility of an intact fibrillin- 1 gene but aberrant transcriptional elements might explain the inability of previous investigators to link known fibrillin-1 gene defects with BAV disease. Although a mutation in genes that regulate transcription has not been associated with congenital aortic valve malformations to date, they are widely implicated in a number of other congenital cardiac anomalies. ${ }^{22}$ BAV disease is a complex and heterogeneous phenotype that might result from genes that encode matrix elements, although it probably does not result from a single gene defect alone, such as the fibrillin-1 gene.

Like the fibrillin-1-deficient mouse, we hypothesized that degeneration of the aortic media in patients with congenital aortic valves is the result of MMP-mediated matrix fragmentation. The MMPs are a family of endogenous enzymes that degrade extracellular matrix components. Increased MMP activity plays an essential role in the formation and progression of aortic aneurysms. ${ }^{23-27}$ Both MMP-2 and MMP-9 are involved in the turnover of elastic matrix components. ${ }^{23}$ MMP-2 is expressed by smooth muscle cells constitutively and might have a pivotal role in the early formation of aneurysms. ${ }^{24-26}$ Crowther and coworkers ${ }^{24}$ established that smooth muscle cells from aneurysmal abdominal aortas produced 3-fold higher levels of MMP-2 than smooth muscle cells from normal aortas. In patients with Marfan syndrome, excessive MMP-2 released from vascular smooth muscle cells is highly localized to areas of matrix fragmentation within the thoracic aortic media, supporting a causative role for MMP-2 in the development of the characteristic vessel wall degeneration. ${ }^{28}$ The role of MMPs in the vascular disease of patients with BAVs had not been previously assessed.

Our observations support the concept, similar to that previously formulated for both atherosclerotic and Marfan aneurysms ${ }^{28}$ that increased MMP activity is involved in the vascular manifestations of congenital BAV disease. Com- 
pared with TAV aortas, MMP-2 activity was increased in the aortas of patients with BAVs (Figure 2), perhaps in response to a reduction in fibrillin-1 microfibrils. Increased MMP activity might indeed disrupt vessel wall integrity, given that MMP-2 activity positively correlated with aortic dilatation (Figure 2,C). Although MMPs appear to mediate aneurysms of diverse causes ${ }^{23}$ the triggers promoting their production and activation might differ. In BAV disease reduced tethering of fibrillin-1 microfibrils from the underlying protein deficiency might activate smooth muscle cells and increase production of MMP-2. In support, pro-MMP-2 was increased in the pulmonary arteries of patients with BAVs, which were also deficient in fibrillin-1. Shear stresses and inflammation might be important activators of the latent MMP-2 produced in the vessel wall, and active MMPs might lead to dilatation. The lower pressures in the pulmonary circulation might have prevented activation of this latent form given that active MMP-2 was not different from controls in the pulmonary artery, and correspondingly, dilatation did not occur in the pulmonary arteries of the patients with BAVs despite the concomitant deficiency of fibrillin-1. These data also suggest that the reduced fibrillin-1 content in the aorta and pulmonary artery is not predominantly the result of MMP degradation, given that fibrillin-1 was reduced in the pulmonary artery without concurrent increases in active MMP species.

Unlike atherosclerotic aortic aneurysms, MMP-9 was not increased in patients with congenital aortic valves. MMP-9 is a major product of macrophages and might be important in atherosclerotic aneurysm formation after prolonged inflammation, particularly in the intermediate to late stages after substantial dilatation. ${ }^{27}$ In the present study aortic dilatation, if present, was only mild to moderate in severity.

\section{Study Limitations}

Because of limitations in sample collection, the study groups were not perfectly matched. In the TAV group, only one patient had significant aortic dilatation, whereas half the patients in the BAV group had significant dilatation. Furthermore, we successfully obtained 6 normal tricuspid valve samples from patients undergoing transplantation; however, all patients in the BAV group had significant valvular disease. Our data must be interpreted in light of these group differences. Second, although fibrillin-1 content was reduced, electron microscopy should be performed in a future study to confirm that fibrillin-1 microfibrils are indeed deficient in the vessel walls of patients with BAVs. Third, the content of other microfibrillar proteins should also be assessed to determine whether bicuspid valve disease is associated with loss of other key microfibrillar matrix components. Fourth, the possibility exists that the pretreatment of tissue samples with elastase led to a nonspecific degradation of fibrillin-1 microfibrils. The disorganized matrix in the
BAV group might have allowed the enzyme to more easily penetrate the tissues of patients with BAVs, resulting in an apparent deficiency of fibrillin-1. However, we used a diluted enzyme concentration and a limited duration of exposure to minimize any nonspecific matrix degradation during elastase treatment. Further studies should be performed to confirm these novel observations.

\section{A Possible Genetic Basis for Aberrant Matrix Remodeling in BAV Disease}

Patients with congenital BAV malformations might have an inherited defect in genes that regulate the processing of microfibrillar proteins into intact stable matrix components in the vessel wall. This might result in a structurally normal protein product but at a significantly reduced amount in the extracellular space. Fibrillin-1 microfibril deficiency might detach smooth muscle cells from the elastic laminae, resulting in matrix degradation and cell death, vessel wall weakening, and progressive aortic dilatation (Figure 4). Aortic aneurysm and dissection might occur when the abundance of fibrillin-rich microfibrils in the media decreases to less than some critical threshold, triggering the release of pathologic MMP species. Although the absence of other associated nonvascular complications is difficult to explain, it might suggest that the clinical phenotype only manifests in areas with high mechanical stress, such as the ascending aorta. As such, under conditions of increased wall stress, a pulmonary autograft in the aortic position in patients with BAV disease might be prone to dilatation given the underlying vascular matrix abnormalities. ${ }^{29}$ The deficient fibrillin- 1 content and increased pro-MMP-2 in the pulmonary artery appears primed for vascular degeneration if subjected to systemic pressures. Secondary events, such as increased wall stress and inflammation, which can activate MMPs that degrade fibrillin-1, could exacerbate the protein deficiency. ${ }^{17}$ The influence of secondary fibrillin- 1 losses over time might explain the phenotypic variability for the aortic dilatation associated with BAVs.

\section{Therapeutic Implications}

Reduced fibrillin-1 content and increased MMP matrix disruption represent novel therapeutic targets to prevent aortic degeneration and dilatation in patients with congenital aortic valve malformations. Therapeutic strategies should perhaps be aimed at blocking secondary events to delay or prevent ongoing matrix degradation. Changes in vessel shear forces can increase MMP-mediated matrix degradation. $\beta$-blockade might minimize shear stresses and limit ongoing matrix remodeling. In addition, perhaps surgical correction of dysfunctional congenital aortic valves should be performed early to afford protection of the aorta rather than waiting and intervening only when necessary to protect the myocardium. Additionally, MMP inhibition can prevent aneurysm formation in animal models. ${ }^{30}$ The role of syn- 


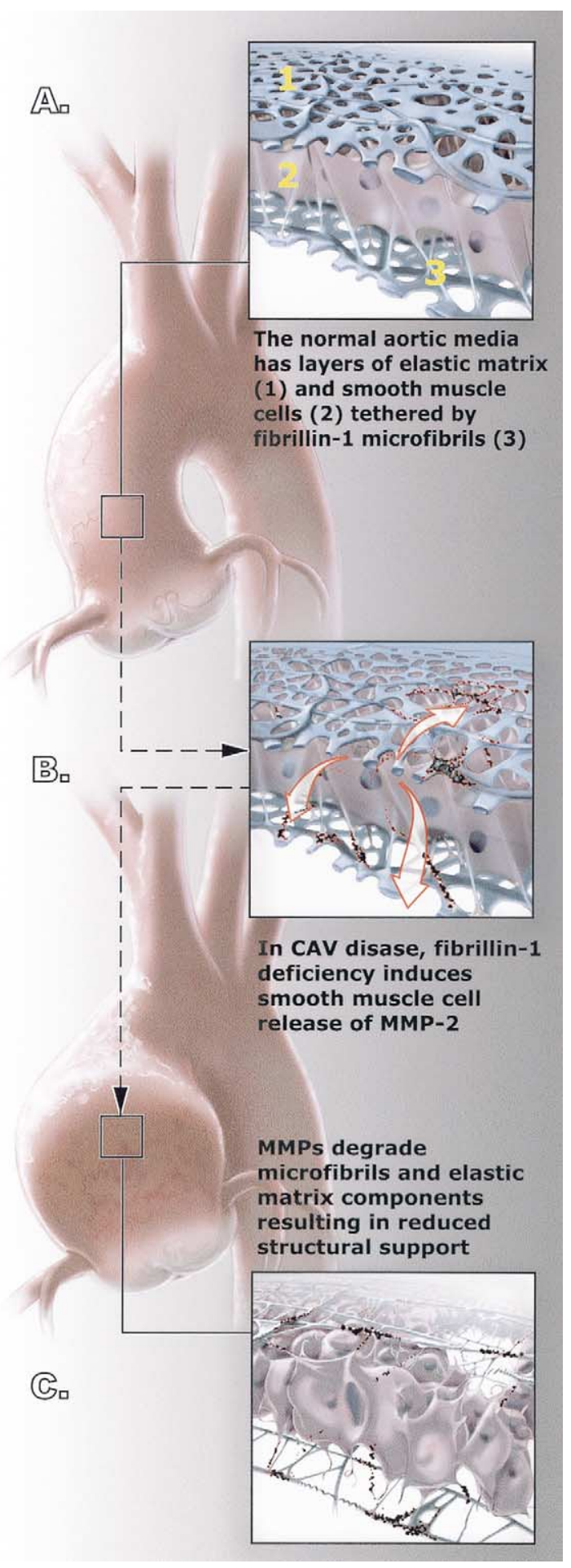

thetic MMP inhibitors to prevent degeneration of the aortic media or gene therapy to replace deficient fibrillin- 1 in patients with this common congenital abnormality might warrant further investigation.
Figure 4. Patients with congenital BAV malformations (CAV) might have an inherited defect in genes that regulate the processing of fibrillin-1 into an intact stable matrix component in the vessel wall. This might result in a structurally normal protein product but at a significantly reduced amount in the extracellular space. Fibrillin-1 microfibril deficiency might detach smooth muscle cells from the elastic laminae, resulting in the release of pathologic MMP species. Released MMP-2 and secondary events, such as increased wall stress and inflammation, that increase MMP activity could weaken the vessel wall by degrading fibrillin-1 and other matrix components. Aortic aneurysm and dissection might occur when the abundance of fibrillin microfibrils in the media decreases to less than some critical threshold, triggering MMP-mediated matrix remodeling and disruption of the vessel wall.

\section{References}

1. Fedak PW, Verma S, David TE, Leask RL, Weisel RD, Butany J. Clinical and pathophysiological implications of a bicuspid aortic valve. Circulation. 2002;106(8):900-4.

2. Dingemans KP, Teeling P, Lagendijk JH, Becker AE. Extracellular matrix of the human aortic media: an ultrastructural histochemical and immunohistochemical study of the adult aortic media. Anat Rec. 2000; 258(1):1-14

3. Bunton TE, Biery NJ, Myers L, Gayraud B, Ramirez F, Dietz HC. Phenotypic alteration of vascular smooth muscle cells precedes elastolysis in a mouse model of Marfan syndrome. Circ Res. 2001;88(1): 37-43.

4. Milewicz DM, Michael K, Fisher N, Coselli JS, Markello T, Biddinger A. Fibrillin-1 (FBN1) mutations in patients with thoracic aortic aneurysms. Circulation. 1996;94(11):2708-11.

5. Braverman AC. Bicuspid aortic valve and associated aortic wall abnormalities. Curr Opin Cardiol. 1996;11(5):501-3.

6. Godfrey M, Nejezchleb PA, Schaefer GB, Minion DJ, Wang Y, Baxter BT. Elastin and fibrillin mRNA and protein levels in the ontogeny of normal human aorta. Connect Tissue Res. 1993;29(1):61-9.

7. Strauss BH, Chisholm RJ, Keeley FW, Gotlieb AI, Logan RA, Armstrong PW. Extracellular matrix remodeling after balloon angioplasty injury in a rabbit model of restenosis. Circ Res. 1994;75(4):650-8.

8. LaBourene JI, Coles JG, Johnson DJ, Mehra A, Keeley FW, Rabinovitch $\mathrm{M}$. Alterations in elastin and collagen related to the mechanism of progressive pulmonary venous obstruction in a piglet model. A hemodynamic, ultrastructural, and biochemical study. Circ Res. 1990; 66(2):438-56.

9. Cruz TF, Mills G, Pritzker KP, Kandel RA. Inverse correlation between tyrosine phosphorylation and collagenase production in chondrocytes. Biochem J. 1990;269(3):717-21.

10. de Sa M, Moshkovitz Y, Butany J, David TE. Histologic abnormalities of the ascending aorta and pulmonary trunk in patients with bicuspid aortic valve disease: clinical relevance to the Ross procedure. J Thorac Cardiovasc Surg. 1999;118(4):588-94.

11. Niwa K, Perloff JK, Bhuta SM, Laks H, Drinkwater DC, Child JS, et al. Structural abnormalities of great arterial walls in congenital heart disease: light and electron microscopic analyses. Circulation. 2001; 103(3):393-400.

12. Ramirez F, Gayraud B, Pereira L. Marfan syndrome: new clues to genotype-phenotype correlations. Ann Med. 1999;31(3):202-7.

13. Pereira L, Lee SY, Gayraud B, Andrikopoulos K, Shapiro SD, Bunton $\mathrm{T}$, et al. Pathogenetic sequence for aneurysm revealed in mice underexpressing fibrillin-1. Proc Natl Acad Sci U S A. 1999;96(7):3819-23.

14. Pereira L, Andrikopoulos K, Tian J, Lee SY, Keene DR, Ono R, et al. Targeting of the gene encoding fibrillin-1 recapitulates the vascular aspect of Marfan syndrome. Nat Genet. 1997;17(2):218-22.

15. Bonderman D, Gharehbaghi-Schnell E, Wollenek G, Maurer G, Baumgartner H, Lang IM. Mechanisms underlying aortic dilatation in congenital aortic valve malformation. Circulation. 1999;99(16):2138-43. 
16. Osakabe T, Okada N, Wachi H, Sato A, Sasaki S, Wada N, et al. Quantitative changes of elastin, fibrillin and collagen in abdominal aortic aneurysms (in Japanese). Nippon Ronen Igakkai Zasshi. 2000; 37(12):979-83.

17. Ashworth JL, Murphy G, Rock MJ, Sherratt MJ, Shapiro SD, Shuttleworth CA, et al. Fibrillin degradation by matrix metalloproteinases: implications for connective tissue remodelling. Biochem J. 1999;340: 171-81.

18. Francke U, Berg MA, Tynan K, Brenn T, Liu W, Aoyama T, et al. A Gly1127Ser mutation in an EGF-like domain of the fibrillin-1 gene is a risk factor for ascending aortic aneurysm and dissection. Am J Hum Genet. 1995;56(6):1287-96.

19. Montgomery RA, Geraghty MT, Bull E, Gelb BD, Johnson M, McIntosh I, et al. Multiple molecular mechanisms underlying subdiagnostic variants of Marfan syndrome. Am J Hum Genet. 1998;63(6):1703-11.

20. Davis EC. Immunolocalization of microfibril and microfibril-associated proteins in the subendothelial matrix of the developing mouse aorta. J Cell Sci. 1994;107(suppl):727-36.

21. Eisenberg LM, Markwald RR. Molecular regulation of atrioventricular valvuloseptal morphogenesis. Circ Res. 1995;77(1):1-6.

22. Epstein JA, Buck CA. Transcriptional regulation of cardiac development: implications for congenital heart disease and DiGeorge syndrome. Pediatr Res. 2000;48(6):717-24.

23. Longo GM, Xiong W, Greiner TC, Zhao Y, Fiotti N, Baxter BT Matrix metalloproteinases 2 and 9 work in concert to produce aortic aneurysms. J Clin Invest. 2002;110(5):625-32.

24. Crowther M, Goodall S, Jones JL, Bell PR, Thompson MM. Increased matrix metalloproteinase 2 expression in vascular smooth muscle cells cultured from abdominal aortic aneurysms. J Vasc Surg. 2000;32(3): 575-83.

25. Davis V, Persidskaia R, Baca-Regen L, Itoh Y, Nagase H, Persidsky $\mathrm{Y}$, et al. Matrix metalloproteinase-2 production and its binding to the matrix are increased in abdominal aortic aneurysms. Arterioscler Thromb Vasc Biol. 1998;18(10):1625-33.

26. Goodall S, Crowther M, Hemingway DM, Bell PR, Thompson MM. Ubiquitous elevation of matrix metalloproteinase-2 expression in the vasculature of patients with abdominal aneurysms. Circulation. 2001; 104(3):304-9.

27. McMillan WD, Tamarina NA, Cipollone M, Johnson DA, Parker MA, Pearce WH. Size matters: the relationship between MMP-9 expression and aortic diameter. Circulation. 1997;96(7):2228-32.

28. Segura AM, Luna RE, Horiba K, Stetler-Stevenson WG, McAllister HA Jr, Willerson JT, et al. Immunohistochemistry of matrix metalloproteinases and their inhibitors in thoracic aortic aneurysms and aortic valves of patients with Marfan's syndrome. Circulation. 1998; 98(suppl 19):II331-7.

29. David TE, Omran A, Ivanov J, Armstrong S, de Sa MP, Sonnenberg $\mathrm{B}$, et al. Dilation of the pulmonary autograft after the Ross procedure. J Thorac Cardiovasc Surg. 2000;119(2):210-20.

30. Allaire E, Forough R, Clowes M, Starcher B, Clowes AW. Local overexpression of TIMP-1 prevents aortic aneurysm degeneration and rupture in a rat model. J Clin Invest. 1998;102(7):1413-20.

\section{Discussion}

Mr Magdi H. Yacoub (London, United Kingdom). I enjoyed your presentation. In the absence of any genomic DNA analysis, how can you say that this is a genetic defect, particularly when stretching is known to activate MMPs and possibly degrades fibrillin-1. Therefore how can you be sure that your findings are not secondary rather than the cause of the problem? Have you matched the sizes of the aorta in your control valves and in your BAVs?

Dr Fedak. Thank you, Mr Yacoub. I hope I have not given the impression that we believe that we have proved that aortic root dilatation in patients with BAVs is the result of a specific gene defect. Our data are suggestive, but there are, as you outlined, other possible explanations for our findings. However, in transgenic mouse models a targeted reduction of aortic fibrillin- 1 con- tent results in a similar sequence of events that includes excessive MMP activity, medial degeneration, and progressive aortic dilatation. In addition, when vessels are subjected to excessive stretching (ie, balloon angioplasty), the arteries characteristically respond with hyperplasia and wall thickening rather than the matrix degradation and dilatation that we have demonstrated in patients with bicuspid valves. These studies and others indicate that matrix degradation is a cause rather than a consequence of aneurysm formation. Shear stresses likely play an important role in exacerbating and maintaining arterial dilatation once it has occurred but probably do not initiate the process. Increased shear stresses alone could not explain why young patients with normally functioning BAVs and normal aortic shear stresses also have significant root dilatation. Further work should be done to identify whether pathologic matrix degradation and deficient fibrillin-1 are indeed the cause of aortic dilatation and whether this process is the result of a specific gene defect. We believe that we have identified an important molecular process that results in aortic dilatation, but our data do not definitively demonstrate a genetic cause. In light of this, I think your measured perspective on our data is very appropriate. We agree with your comments, and that is why in our abstract title we used the phrase "a possible gene defect." We also revised the title of our article to minimize potential confusion.

Dr Thoralf M. Sundt (Rochester, Minn). I agree with the professor and in fact would argue that your correlation between aortic diameter and MMPs argues exactly this point. What you have observed could well be a secondary biomechanical response to alterations in transmural stress distribution occurring with dilatation rather than a reflection of a primary genetic abnormality.

I would like to note that there is more to the phenotype of ascending aortic aneurysms associated with bicuspid valves than you imply in your presentation. There is not just one phenotype but in fact a number of different kinds of ascending aortic aneurysms associated with bicuspid valves: some aneurysms arise entirely above the sinotubular ridge with normal sinuses and, commonly, aortic stenosis. There is also another configuration that looks for all the world like Marfan syndrome with dilatation of the sinuses, as well as the ascending aorta, and an onion-shaped root with, most often, aortic regurgitation. Do you have any more precise information about the phenotypes of the individuals that you were studying?

Dr Fedak. In my presentation I outlined that some patients with BAVs had normal aortic fibrillin-1 content. Clearly, not every patient with BAV disease is equivalent on a molecular level, and as you outlined, this is also true on a clinical level. Some younger patients with aortic insufficiency manifest aortic dilatation early, and some older patients with stenosis never have significant aortic dilatation. These diverse clinical phenotypes combined with our heterogeneous molecular data suggest that if there are genes involved, and I believe that there are, likely there is more than one involved. I believe that a host of genes, all probably encoding matrix components, are involved in this disease and result in diverse clinical phenotypes. Unfortunately, our small sample size precluded a useful analysis of these important issues.

Dr Frank W. Sellke (Boston, Mass). That was sort of my question. What is known about the genetic regulation of fibrillin-1 expression? And the second point was, if you try to inhibit MMP activity and expression, what kind of consequence might you 